\title{
Reaction Mechanism of the Reduction of Ozone on Graphite
}

Eduardo Humeres, Karen M. De Castro, Nito Angelo Debacher, Regina de F. P. M. Moreira

INDEX

Figure S1. X-ray diffraction analysis at different times of $\mathrm{G}_{106 \mu \mathrm{m}}$ ozonized at $100^{\circ} \mathrm{C}$.

Figure S2. First-order rate plot of the insertion of $\mathrm{O}_{3}$ in $\mathrm{G}_{106}$ at $100{ }^{\circ} \mathrm{C}$.

Figure S3. Eyring plot of the ozonation reaction of graphite $\mathrm{G}_{106}$ from $\boldsymbol{k}_{\boldsymbol{p} \boldsymbol{s} \boldsymbol{O}_{3}}$.

Figure S4. Decarboxylation reaction of ozonized $\mathrm{G}_{106}$ at $600{ }^{\circ} \mathrm{C}$.

Figure S5. X-ray diffractogram of graphite $\mathrm{G}_{106}$ after total deoxygenation.

Figure S6. Elimination of oxygen during the ozonation of $\mathrm{G}_{6.2}$ at $100{ }^{\circ} \mathrm{C}$.

Figure S7. FTIR spectrum of grafhite $\mathrm{G}_{106 \mu \mathrm{m}}$ upon ozonation at $50^{\circ} \mathrm{C}$.

Figure S8. FTIR spectrum of grafhite $\mathrm{G}_{106 \mu \mathrm{m}}$ upon ozonation at $100{ }^{\circ} \mathrm{C}$.

Figure S9. FTIR spectrum of grafhite $\mathrm{G}_{106 \mu \mathrm{m}}$ after partial decarboxylation at $600{ }^{\circ} \mathrm{C} . \quad \mathbf{0 6}$

Figure S10. FTIR spectrum of ozonized $\mathrm{G}_{106 \mu \mathrm{m}}$ after total decarboxylation.

Table S1. Thermal annealing of $\mathrm{G}_{106}$

Table S2. FTIR absorption bands and assignments for oxidized carbonaceous materials. 


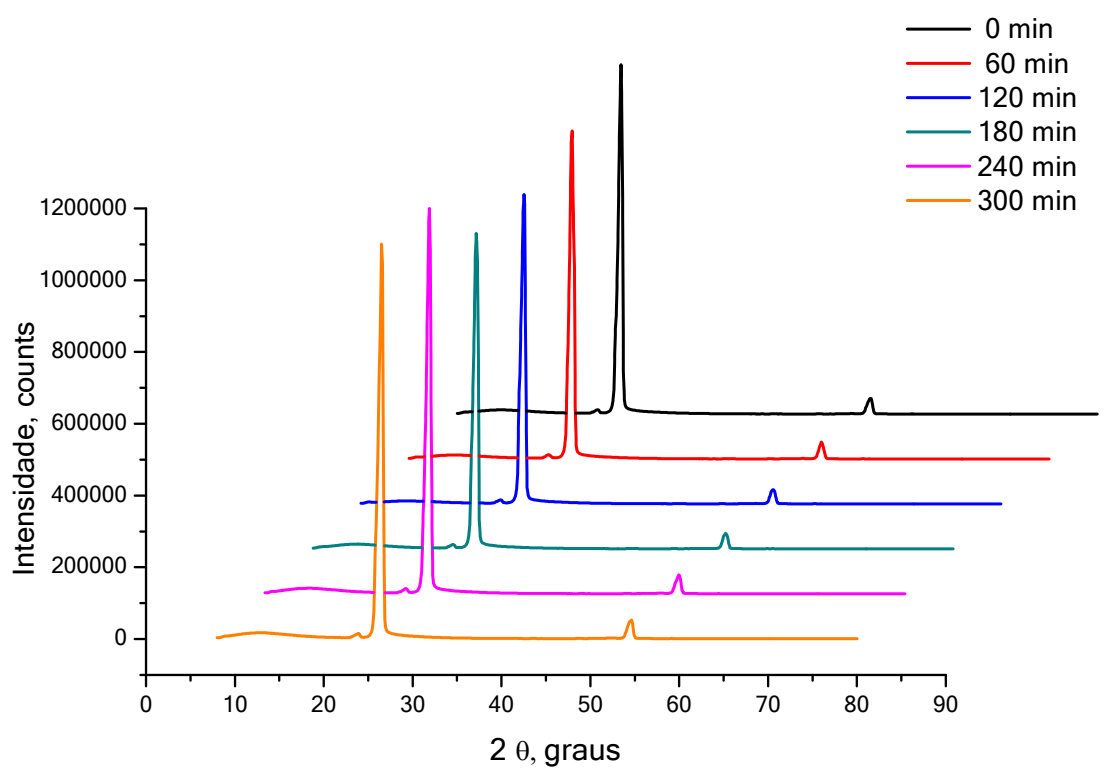

Figure S1. X-ray diffraction analysis at different times of $\mathrm{G}_{106 \mu \mathrm{m}}$ ozonized at $100{ }^{\circ} \mathrm{C}$.

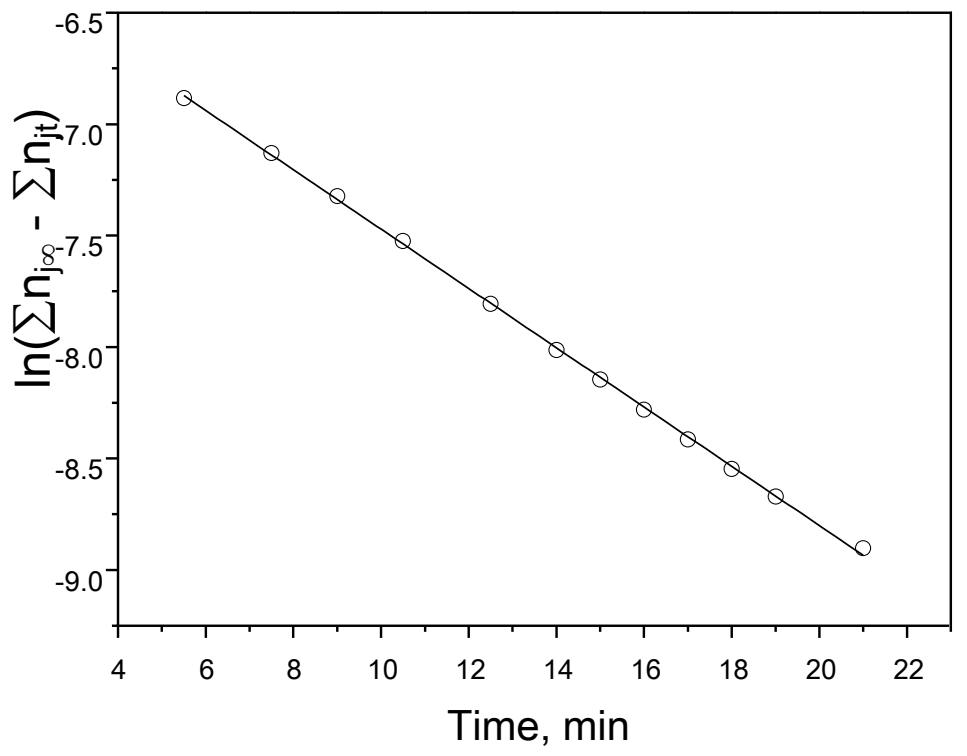

Figure S2. First-order rate plot of the insertion of $\mathrm{O}_{3}$ in $\mathrm{G}_{106}$ at $100{ }^{\circ} \mathrm{C} ;\left[\mathrm{O}_{3}\right] 5.12 \times 10^{-4} \mathrm{~mol} \cdot \mathrm{L}^{-1} ; \mathrm{O}_{2}$ flow $0.60 \mathrm{~L} \cdot \mathrm{min}^{-1}$; sample $3.00 \mathrm{~g}$ 


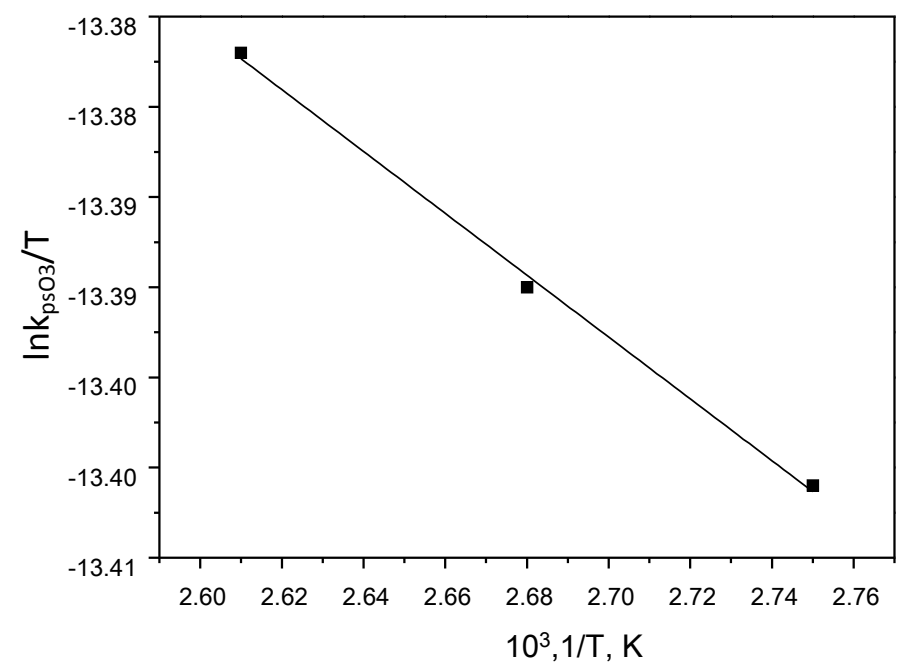

Figure S3. Eyring plot of the ozonation reaction of graphite $\mathrm{G}_{106}$ from $\boldsymbol{k}_{\boldsymbol{p s}} \boldsymbol{o}_{3}$.

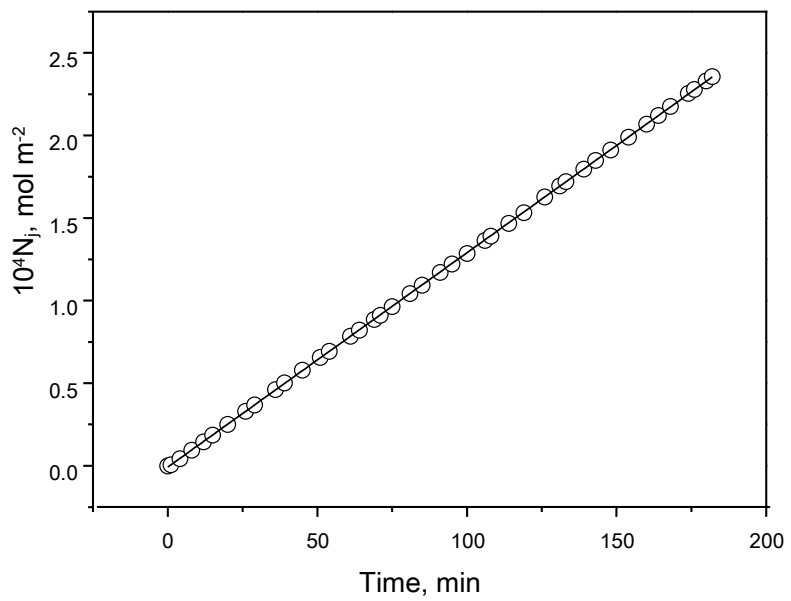

Figure S4. Decarboxylation reaction of ozonized $\mathrm{G}_{106}$ at $600{ }^{\circ} \mathrm{C}$; sample $2.46 \mathrm{~g}$; He flow 40 $\mathrm{ml} \cdot \mathrm{min}^{-1}$. 


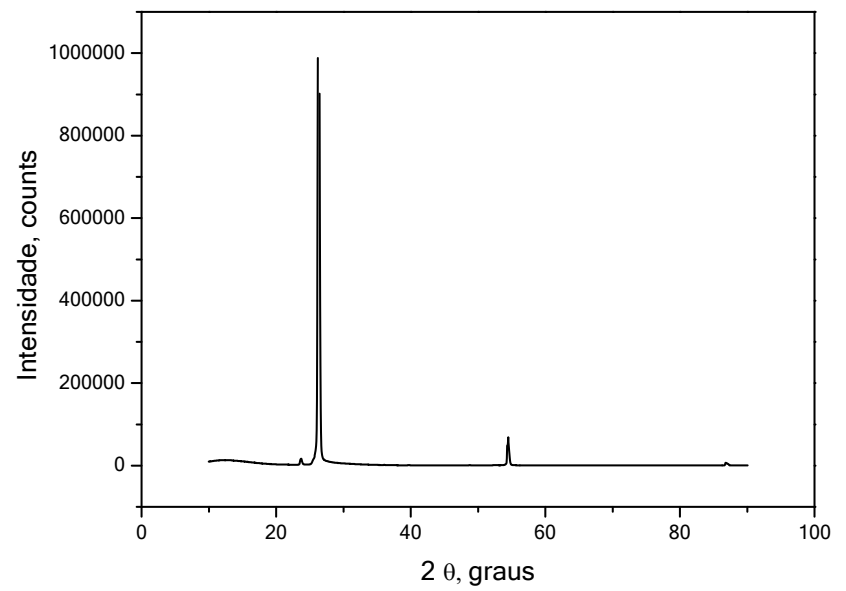

Figure S5. X-ray diffractogram of graphite $\mathrm{G}_{106}$ after total deoxygenation.

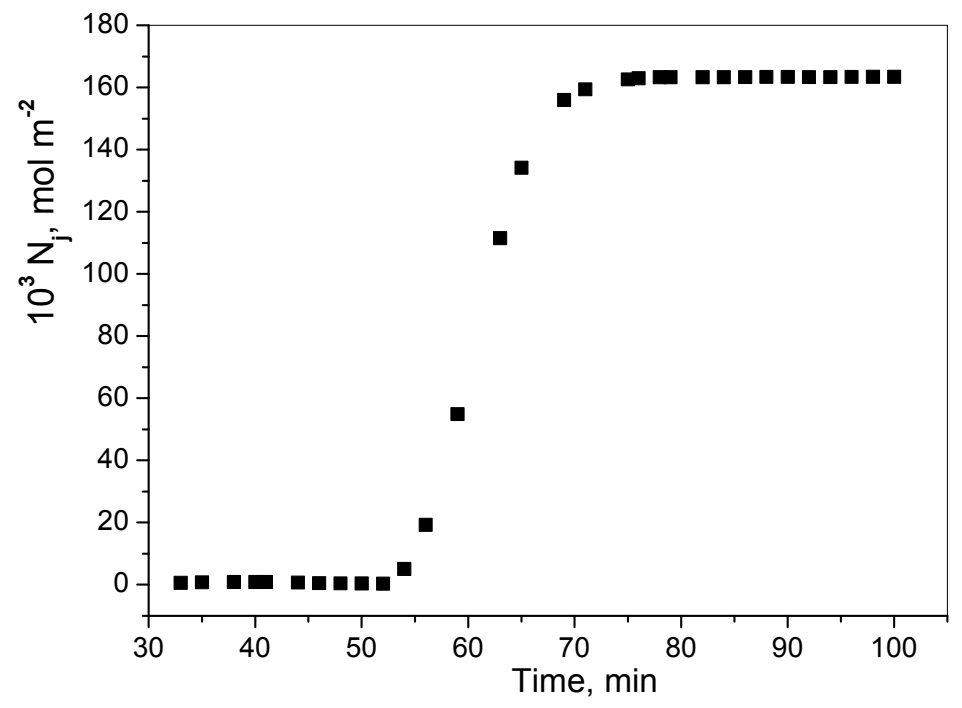

Figure S6. Elimination of oxygen during the ozonation of $\mathrm{G}_{6.2}$ at $100{ }^{\circ} \mathrm{C} ;\left[\mathrm{O}_{3}\right] 5.17 \times 10^{-4} \mathrm{~mol} \cdot \mathrm{L}^{-1}$; solid $1.40 \mathrm{~g} ; \mathrm{O}_{2}$ flow $0.60 \mathrm{~L} \cdot \mathrm{min}^{-1}$. 


\section{FTIR SPECTRA}

The FTIR spectra were obtained on a Perkin Elmer FTIR-100 single path spectrometer.

From air: $\mathrm{H}_{2} \mathrm{O}\left(4333 \mathrm{~cm}^{-1}\right) ; \mathrm{CO}_{2}\left(2380 \mathrm{~cm}^{-1}, 1675 \mathrm{~cm}^{-1}\right)$.

Table S2 shows the FTIR absorption bands and assignments for oxidized carbonaceous materials.

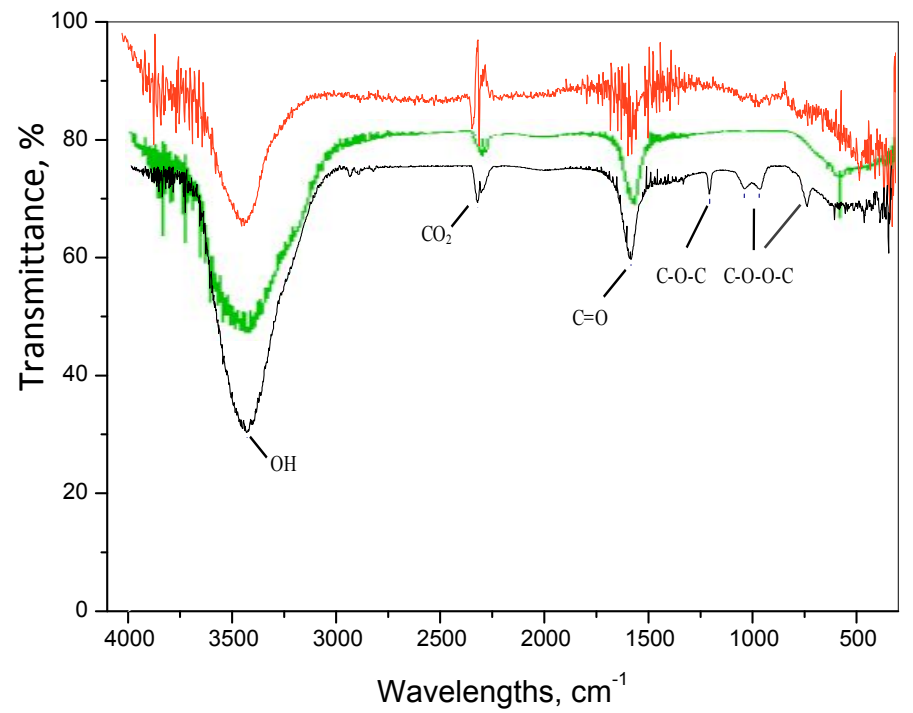

Figure S7. FTIR spectrum of grafhite $\mathrm{G}_{106 \mu \mathrm{m}}$ upon ozonation at $50{ }^{\circ} \mathrm{C}$. Red, before ozonation; green, $10 \mathrm{~min}$; black, $120 \mathrm{~min}$.

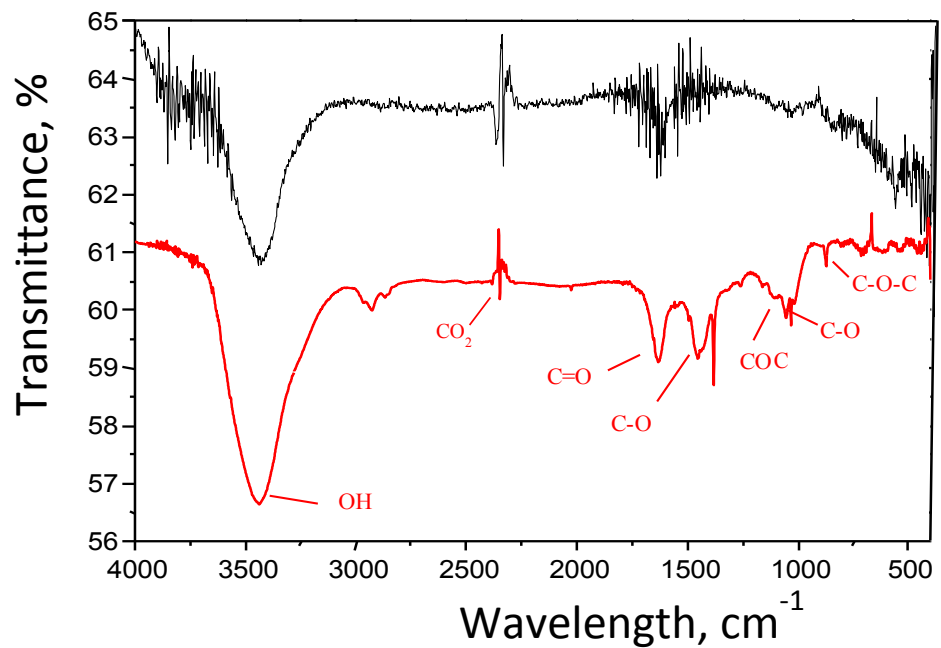

Figure S8. FTIR spectrum of grafhite $\mathrm{G}_{106 \mu \mathrm{m}}$ upon ozonation at $100^{\circ} \mathrm{C}$. Black, before ozonation; red, $120 \mathrm{~min}$. 


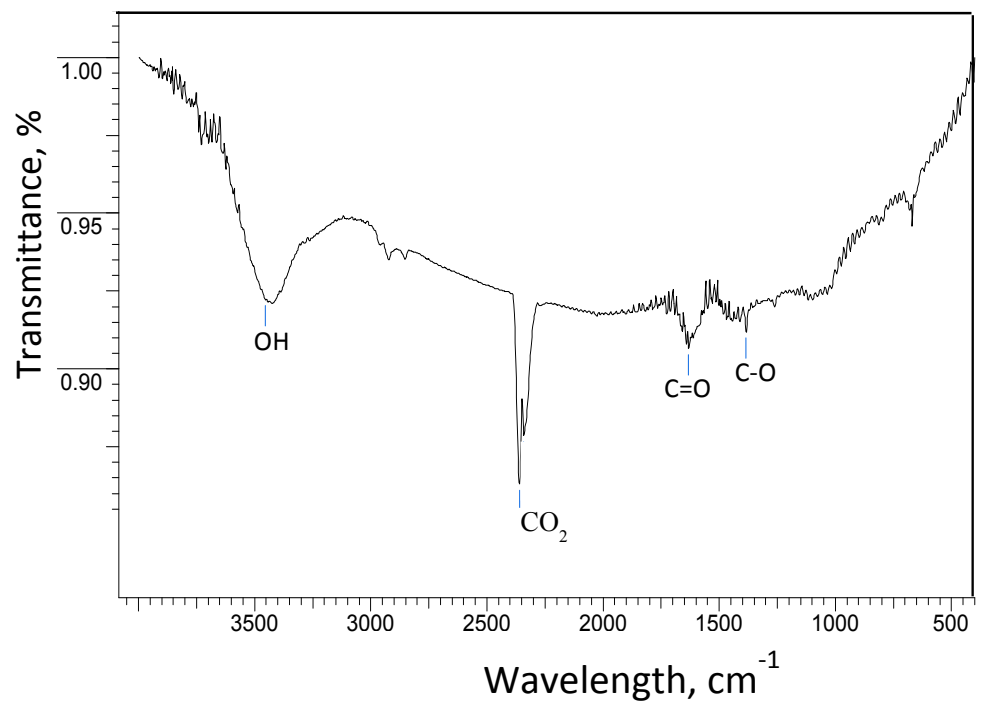

Figure S9. FTIR spectrum of grafhite $\mathrm{G}_{106 \mu \mathrm{m}}$ after partial decarboxylation at $600{ }^{\circ} \mathrm{C}$.

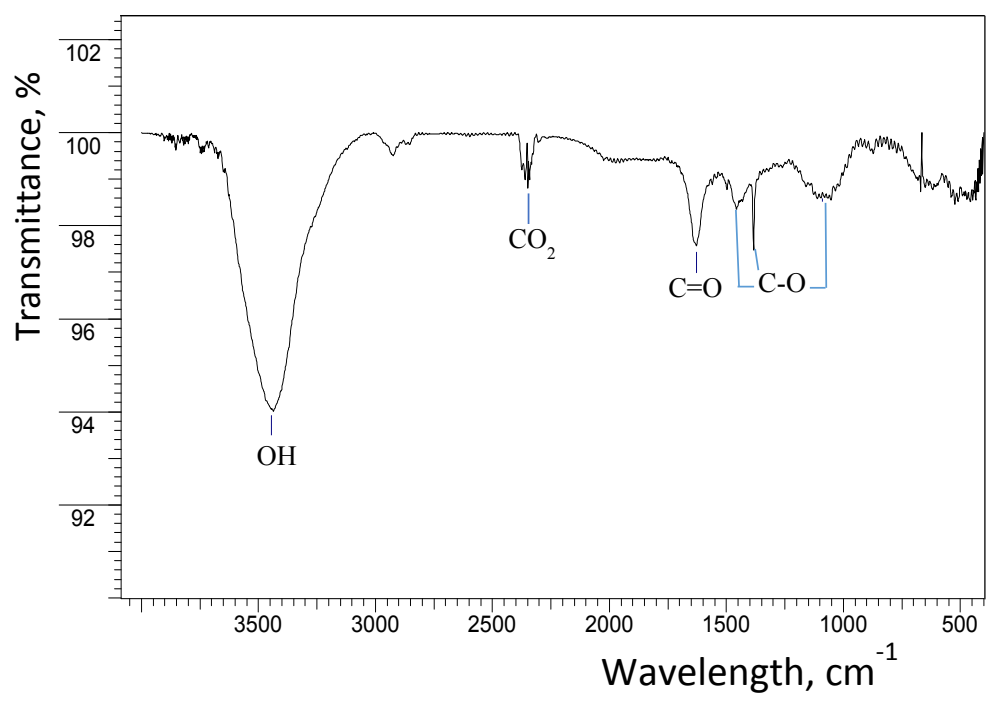

Figure S10. FTIR spectrum of ozonized $\mathrm{G}_{106 \mu \mathrm{m}}$ after total decarboxylation. 
Table S1. Thermal annealing of $\mathrm{G}_{106}$.

\begin{tabular}{|c|c|c|}
\hline Temperature, ${ }^{\circ} \mathrm{C}$ & Time, $\min$ & Specific area,${ }^{\mathrm{a}} \mathrm{m}^{2} \cdot \mathrm{g}^{-1}$ \\
\hline- & - & $1.30^{\mathrm{b}}$ \\
100 & 90 & $2.58^{\mathrm{c}}$ \\
300 & 15 & 4.67 \\
820 & 4 & 2.90 \\
820 & 20 & 2.57 \\
820 & 240 & 1.95 \\
820 & 360 & 0.26 \\
1000 & 5 & 3.23 \\
\hline
\end{tabular}

${ }^{a} \mathrm{~N}_{2}$ used as adsorbate. ${ }^{b}$ Pristine sample. ${ }^{\mathrm{c}} \mathrm{After}$ ozonation.

Table S2. FTIR absorption bands and assignments for oxidized carbonaceous materials.

\begin{tabular}{|c|c|c|c|}
\hline $\begin{array}{l}\text { Functional } \\
\text { Group }\end{array}$ & Assignment & $\begin{array}{c}\text { wavenumber, } \\
\text { cm }^{-1}\end{array}$ & References \\
\hline \multirow{4}{*}{$\mathrm{C}-\mathrm{O}$} & alcohol & $1030-1094$ & $01 ; 02 ; 03$ \\
\hline & -C-O-O-C-, epoxide, ozonide & $1250-1040$ & $04 ; 05 ; 06 ; 07$ \\
\hline & tertiary alcohols & 1450,1386 & $08 ; 02 ; 03$ \\
\hline & ozonides, ethers, esters, lactone & $1100-1250$ & $09 ; 01 ; 08$ \\
\hline \multirow{2}{*}{ C-O-C } & bending (epoxide)) & $800-900,980,992$ & $09 ; 10 ; 03$ \\
\hline & stretching (epoxide) & $1200-1300$ & $09 ; 01 ; 08 ; 03$ \\
\hline \multirow{6}{*}{$\mathbf{O H}$} & bending (absorbed water) & $1625-1640$ & $09 ; 08 ; 02 ; 03$ \\
\hline & stretching (free and absorbed water) & $3028,3400-3600$ & $08 ; 02 ; 11 ; 03$ \\
\hline & $\begin{array}{l}\text { stretching (absorbed water and } \\
\text { carboxylic acids) }\end{array}$ & $3100-3700$ & $09 ; 12 ; 13 ; 03$ \\
\hline & carboxylic acid stretching & 1710,1730 & 11 \\
\hline & $\mathrm{C}-\mathrm{OH}$ & $1340-1384$ & 02 \\
\hline & aromatic stretching & 1224 & 10 \\
\hline \multirow{5}{*}{$\mathbf{C}=\mathbf{O}$} & stretching & 1634 & 14 \\
\hline & stretching of carboxylic acid & $1710-1760$ & $13 ; 09 ; 01 ; 08 ; 12 ; 10 ; 02$ \\
\hline & carboxylic acid & 1635 & $15 ; 14 ; 16$ \\
\hline & carbonyl & $1365-1383$ & 11 \\
\hline & ketone stretching & 1705,1680 & 14 \\
\hline $\mathrm{C}=\mathrm{C}$ & stretching & $1537-1630$ & $09 ; 01 ; 08 ; 12 ; 10$ \\
\hline $\mathrm{CO}_{2}$ & stretching & 2336 & 09 \\
\hline \multirow[b]{2}{*}{ C-H } & aromatic & 3060 & 11 \\
\hline & $\begin{array}{c}\text { stretching } \mathrm{C}-\mathrm{H} \text { bonds influenced by } \\
\mathrm{O}_{3} \text { treatment }\end{array}$ & $2855-2925$ & 08 \\
\hline $\mathrm{C}-\mathrm{C}$ & stretching & 1550,1168 & $02 ; 08$ \\
\hline $\mathrm{COOH}$ & stretching & 1710 & 11 \\
\hline
\end{tabular}




\section{FTIR references}

S1. Si, Y.; Samulski, E. T. Synthesis of Water-Soluble Graphene. Nano Letters, 2008, 8, 16791682.

S2. Khai, T. V.; Na, H. G.; Kwak, D. S.; Kwon, Y. J.; Ham, H.; Shim, K. B.; Kim, H. W. Influence of N-doping on the Structural and Photoluminescence Properties of Graphene Oxide Films. Carbon, 2012, 50, 3799-3806.

S3. Mermoux. M.; Chabre, Y.; Rousseau. A. FTIR and 13C NMR Study of Graphite Oxide. Carbon, 1991, 29, 469-474.

S4. Krawczyk, P.; Skowronski, J. M. Modification of Expanded Graphite Electrodes by Ozone Treatment. Acta Phys. Pol. A, 2010, 118, 465-470.

S5. Peebles, B. C.; Dutta, P. K.; Waldman, W. J.; Villamena, F. A.; Nash, K.; Severance, M.; Nagy, A. Physicochemical and Toxicological Properties of Commercial Carbon Blacks Modified by Reaction with Ozone. Environ. Sci. Technol. 2011, 45, 10668-10675,

S6. Lee, D. W.; L. Santos, V. D. L.; Seo, J. W.; Felix, L. L.; Bustamante, A. D.; Cole, J. M.; Barnes, C. H. W. The Structure of Graphite Oxide: Investigation of it Surfaces Chemical Groups. J. Phys. Chem. B, 2010, 114, 5723-5728.

S7. Mawhinney, D. B.; Yates, J. T. J. FTIR Study of the Oxidation of Amorphous Carbon by Ozone at $300 \mathrm{~K}$ - Direct COOH Formation. Carbon. 2001, 39, 1167-1173.

S8. Wang. H.; Hu, Y.H. Effect of Oxygen Content on Structures of Graphite Oxides. Ind. Eng. Chem. Res. 2011, 50, 6132-6137.

S9. Eigler, S.; Dotzer, C.; Hirsch, A.; Enzelberger, M.; Müller, P. Formation and Decomposition of $\mathrm{CO}_{2}$ Intercalated Graphene Oxide. Chem. Mater. 2012, 24, 1276-1282.

S10. Liao, S. H.; Liu, P. L.; , Hsiao, M. C.; Teng, C. C.; Wang, C. A.; Ger, M. D.; Chiang, C. L. One-Step Reduction and Functionalization of Graphene Oxide with Phosphorus-Based Compound to Produce Flame-Retardant Epoxy Nanocomposite. Ind. Eng. Chem. Res. 2012, 51, 4573-4581.

S11. Silverstein, R. M.; Webster, F. X.; Kiemle, D. J. Identificação Espectrofotométrica de Composto Orgânicos. $7^{\text {th }}$ Ed. Rio de Janeiro: LTC, 2011. 
S12 Pham, P. A.; Kumar, N. A.; Jeong, Y. T. Covalent Functionalization of Graphene Oxide With Polyglycerol and Their Use as Templates for Anchoring Magnetic Nanoparticles. Synth. Met. 2010, 160, 2028-2036.

S13 Cao, H.; Wu, X.; Yin, G.; Warner, J. H. Synthesis of Adenine-Modified Reduced Graphene Oxide Nanosheets. Inorg. Chem. 2012, 51, 2954-2960.

S14 Paiva, D. L.; Lampman, G. M.; Kriz, G. S.; Vyvyan, J. R. Introdução à espectroscopia. Portuguese Ed. from $4^{\text {th }}$ English Ed., São Paulo: Cengage Learning, 2010.

S15 Chang, C. H. Preparation and Characterization of Carbon-Sulfur Surface Compounds. Carbon, 1981, 19, 175-186.

S16 Hontoria-Lucas, C.; López-Peinado, A. J.; López-González, J. D.; Rojas-Cervantes, M. L.; Martín-Aranda, R.M. Study of Oxygen-Containing Groups in a Series of Graphite Oxides: Physical and Chemical Characterization. Carbon, 1995, 33, 1585- 1592. 